\title{
Ultra-low friction and excellent elastic recovery of fullerene-like hydrogenated carbon film based on multilayer design
}

\author{
YANSHUANG MENG ${ }^{1}$, XIAOLONG JIA ${ }^{1,2}$, FULIANG ZHU ${ }^{1, *}$ and JUNYAN ZHANG ${ }^{2}$ \\ ${ }^{1}$ School of Material Science and Engineering, Lanzhou University of Technology, Lanzhou 730000, China \\ ${ }^{2}$ State Key Laboratory of Solid Lubrication, Lanzhou Institute of Chemical Physics, Chinese Academy of Sciences, \\ Lanzhou 730000, China \\ *Author for correspondence (chzfl@126.com)
}

MS received 18 October 2016; accepted 13 December 2016; published online 24 August 2017

\begin{abstract}
Multilayer fullerene-like hydrogenated carbon (FL-C:H) films were synthesized by using the chemical vapour deposition technique with a different flow rate of methane. The typical fullerene-like structure of as-prepared films was investigated by using transmission electron microscopy and Raman spectra. The prepared multilayered FL-C:H films showed a high elastic recovery $(\sim 90 \%)$, ultra-low friction coefficient $(\sim 0.019)$ and low wear rate $\left(\sim 3.0 \times 10^{-9} \mathrm{~mm}^{3} \mathrm{Nm}^{-1}\right)$ in humid air.
\end{abstract}

Keywords. Carbon films; fullerene-like structure; elastic recovery; super-lower wear rate.

\section{Introduction}

In recent years, the fullerene-like hydrogenated carbon (FL$\mathrm{C}: \mathrm{H})$ films have been the focus of an ever increasing amount of research as a promising super-lubrication material [1,2]. The fullerene (FL) structured films have been synthesized by a variety of methods including magnetron sputtering [3], low energy ion beam deposition [4] and pulse plasma-enhanced chemical vapour deposition (PECVD) [5]. Of these methods, pulse-PECVD is considered the best candidate because the pulse power facilitates bombardment of energetic ions, which promotes the formation of FL nanostructures [6]. Since the FL structure is composed of curved and cross-linked graphite sheets in a three-dimensional arrangement, films made by this FL structure exhibit extremely low friction coefficient, prominent wear resistance and high friction stability under constant sliding at high pressure [5,6]. Recent studies in nanoscale and macroscale showed that the FL structures achieve super-low friction based on the theory of incommensurate contact sliding against dual ball [7]. Since the FL structure can reserve the elastic energy during distortion through reversible bond rotation and bond angle deflection, the FL films display both ideal stiffness and flexibility while reducing friction [8]. In addition, curved graphite sheets can significantly reduce the energetic dangling at the edge of the structure, which weakens the interactions at sliding faces, therefore, an extremely low friction is achieved [6].

On the other side, many researchers have reported that annealing treatment [9], using various bias voltage [10] and adding the catalyst $[11,12]$ will have a significant impact on FL structure. Many researchers have demonstrated that the FL structure was dependent on the synthesizing conditions, such as annealing treatment [9], bias voltage during chemical vapour deposition [10] and catalyst [11,12]. In our previous work, we successfully fabricated various monolayer FL structure films with excellent performance by using the PECVD technology $[5,8]$. Recently, the multilayer hydrogenated amorphous carbon (a-C:H) film is gaining increased interest since the multilayered structure can effectively reduce stress $[13,14]$, improve hardness $[15,16]$ and reduce friction $[17,18]$. Therefore, a multilayered nanostructure arranged in a two-dimensional direction is more desired than a simple mixture with carbon matrix.

In this work, we have produced FL-C:H films with a multilayered structure by using the pulse-PECVD method. The novel structure was first characterized by transmission electron microscopy and Raman spectra to determine the quality. Then the mechanical and tribological performance was measured.

\section{Experimental}

\subsection{Films deposition}

The pulse-PECVD method was applied to obtain multilayer FL-C:H films on Si (100) substrates. The initial pressure of the vacuum chamber was pumped to less than $9.9 \times$ $10^{-4} \mathrm{~Pa}$. Silicon wafers were first cleaned in ethanol by ultrasonic bath for $10 \mathrm{~min}$ before putting them into the vacuum chamber. After dying in air, the cleaned silicon wafers were etched at a bias voltage of $-1000 \mathrm{~V}$ and an argon ion plasma pressure of $6.1 \mathrm{~Pa}$ for $30 \mathrm{~min}$ to remove the native oxide surface of silicon. The films were deposited 
Table 1. Deposition parameters.

\begin{tabular}{lccccc}
\hline Sample & S1 & M1 & M2 & M3 & S2 \\
\hline Pressure (Pa) & 30 & $30 / 51$ & $30.5 / 54$ & $30.5 / 54$ & 54.5 \\
Current (A) & 0.37 & $0.31 / 0.40$ & $0.28 / 0.38$ & $0.3 / 0.4$ & 0.47 \\
Thickness & 1.406 & 1.661 & 1.708 & 1.91 & 2.133 \\
$\quad(\mu \mathrm{m})$ & & & & & \\
\hline
\end{tabular}

at a power voltage of $-1000 \mathrm{~V}$, frequency of $79.6 \mathrm{kHz}$ and duty-cycle of $60 \%$ for $2 \mathrm{~h}$. Monolayer film samples were obtained by using a methane flow rate of $30 \mathrm{sccm}$ and 60 sccm and were labelled as S1, S2, respectively. The multilayer films were also synthesized by using a methane flow rate of $30 \mathrm{sccm}$ and $60 \mathrm{sccm}$. According to the time ratio of the multilayer film, samples were labelled as M1 (4/2 min), M2 (4/4 min) and M3 (4/6 min), respectively. The layer number for M1, M2 and M3 is 40, 30 and 24, respectively. The detailed deposition conditions are summarized in table 1.

\subsection{Characterization of films}

The sample thickness was measured by using the crosssectional scanning electron microscope (SEM) images (JEOL JSM-6701F and S-4800). The structure of the FL-C:H films were observed by high-resolution transmission electron microscopy (HRTEM, JEOL 2010) operated at $200 \mathrm{kV}$. The HRTEM samples were obtained by depositing the films on a $\mathrm{NaCl}$ substrate followed by dissolving the $\mathrm{NaCl}$ substrate with water. Micro-Raman backscattering spectra of the samples were recorded on a LabRam HR800 spectrometer (HORIBA Jobin Yvon, France) with Ar laser as the excitation source at $532 \mathrm{~nm}(2.3 \mathrm{eV})$. Laser power was carefully controlled to avoid any heating damage to the samples. Nanoindentation tests of the samples were tested (XP) using a computercontrolled Hysitron Ub1 nanoindenter with depth limit of 150 $\mathrm{nm}$ as a unity. The films were tested on the surface approach sensitivity as $40 \%$. All nanoindentation tests were carried out at room temperature $\left(20^{\circ} \mathrm{C}\right)$ with a relative humidity of $30 \%$. A total of nine indentations were performed for each film. An elastic recovery of the samples can be determined qualitatively by using the indentation load-unload curve. The elastic recovery was calculated by using the following formula (equation 1) [19]:

$$
R=\frac{d_{\text {max }}-d_{\text {res }}}{d_{\text {res }}} \times 100 \%
$$

Where, $R$ is the elastic recovery, $d_{\max }$ is the displacement at maximum load and $d_{\text {res }}$ is the residual or plastic displacement after removing the load. Friction test was conducted using a ball-on-disc reciprocating the tribometer (MFT-R4000). All tests were performed at room temperature with a relative humidity of $40-50 \%$. The mating balls $(5 \mathrm{~mm}$ steel ball) were cleaned with acetone in an ultrasonic cleaner before each test. The reciprocating amplitude was $5 \mathrm{~mm}$ and the average sliding speed was $100 \mathrm{~mm} \mathrm{~s}^{-1}$. The corresponding wear scar morphologies of the samples were observed on a MAX3D surface profiler and the wear volume of the samples was calculated by measuring the crosssectional area at three different locations along the wear track.

\section{Result and discussion}

\subsection{HRTEM and cross-sectional SEM images}

Figure 1a and $\mathrm{b}$ shows the HRTEM images of samples $\mathrm{S} 1$ and $\mathrm{S} 2$, respectively. The long-range order patterns of S1 and S2 can be clearly observed, indicating the existence of the FL nanostructures in the film $[2,20]$. Moreover, the layer spacing of $\sim 0.34 \mathrm{~nm}$, which is the same as the interlayer spacing of graphite [20], further confirms the existence of fullerene-like structure in the films.

The cross-sectional SEM images of the films are shown in figure 2. The films appear to be very dense without any defects. SEM images of monolayer samples S1 and S2, seen in figure $2 \mathrm{a}$ and $\mathrm{b}$, show an average thickness of $\sim 1.406$ and $\sim 2.137 \mu \mathrm{m}$, respectively. The cross-section of the multilayer samples M2 and M3 exhibits layers of various thickness (figure $2 \mathrm{c}$ and $\mathrm{d}$ ), suggesting that the growth rate is low at the beginning, increases in the middle and slows down in the end. When compared with M3, M2 displays more layers, which is due to the experiment design.

\subsection{Raman spectra}

Raman spectrum is widely used to study the quality of carbon films. The Raman spectra of the samples are shown in figure 3a. For the diamond-like carbon (DLC) film, the Raman spectra can be fitted by two Gaussian peaks at about $1580 \mathrm{~cm}^{-1}$ (G peak) and $1350 \mathrm{~cm}^{-1}$ (D peak). The $\mathrm{G}$ peak is due to the bond stretching of $s p^{2}$ atoms in both aromatic rings

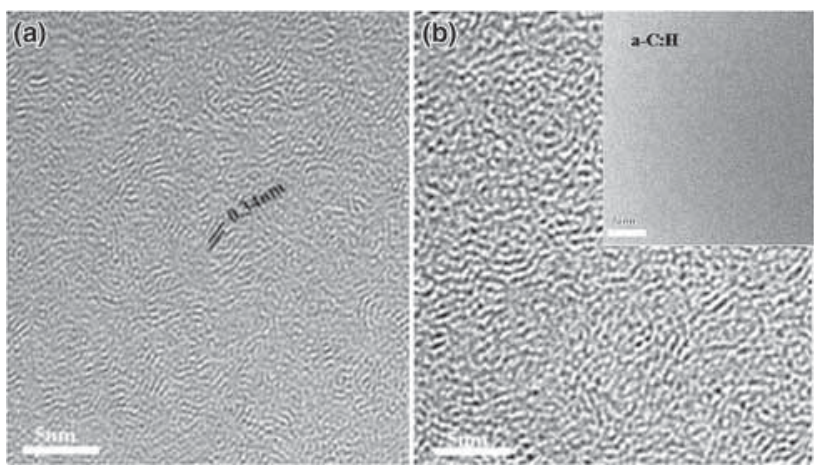

Figure 1. HRTEM images of the as-prepared samples: (a) film S1 and (b) film S2. 

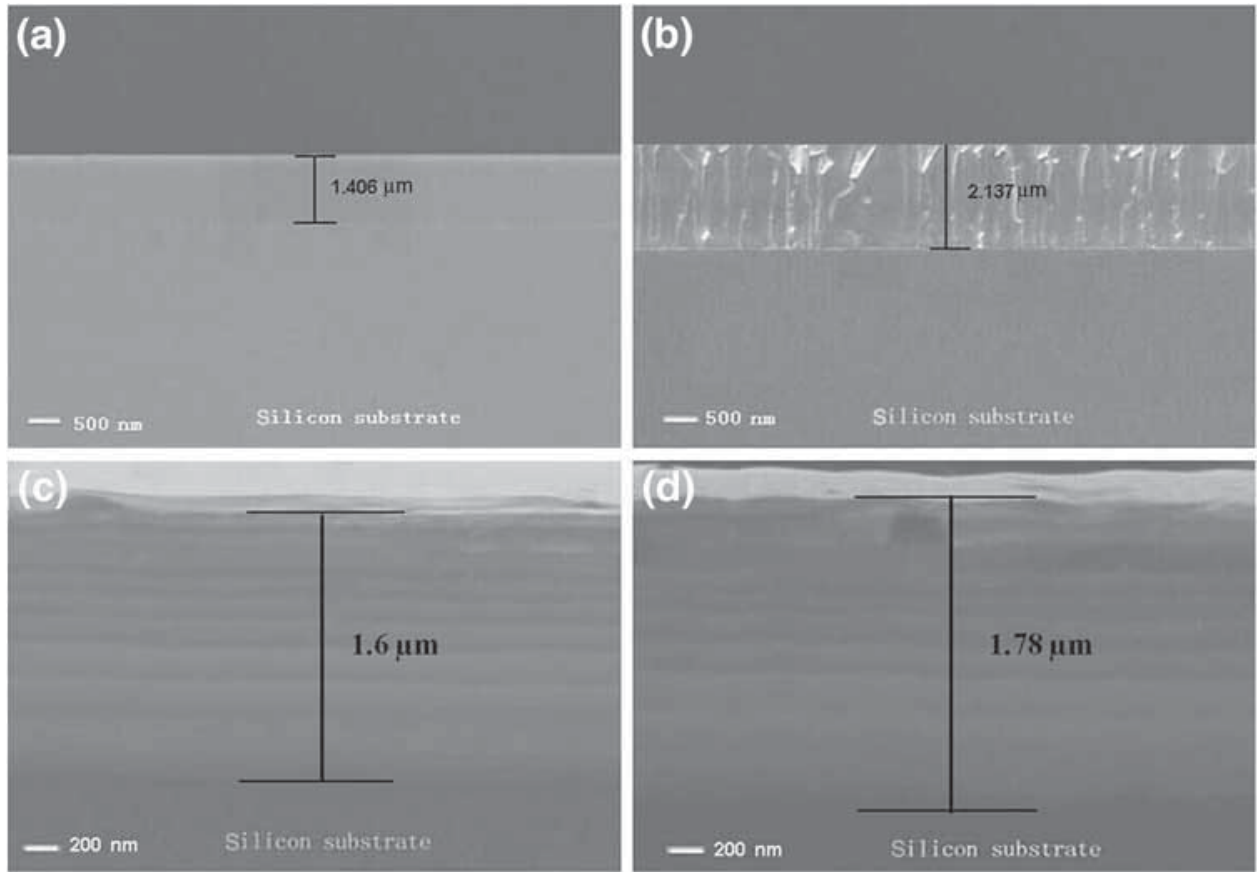

Figure 2. SEM cross-section images of monolayer (a) S1 film, (b) S2 film and multilayer (c) M2 film and (d) M3 film.

(a)

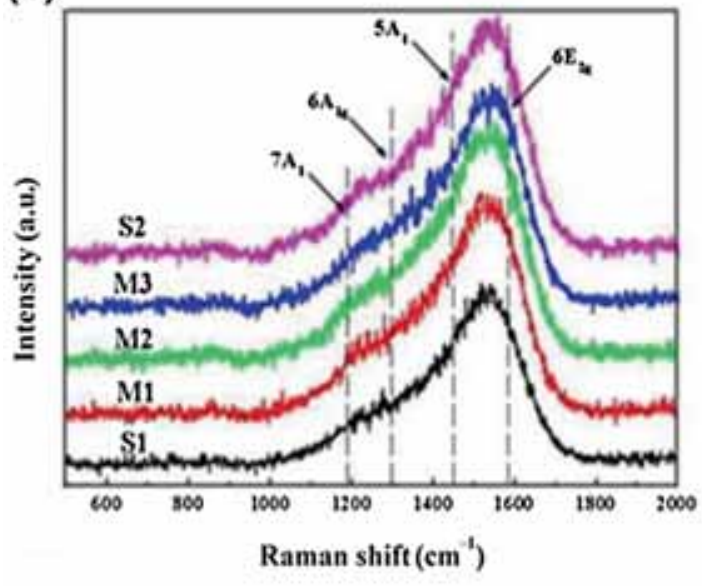

(b)

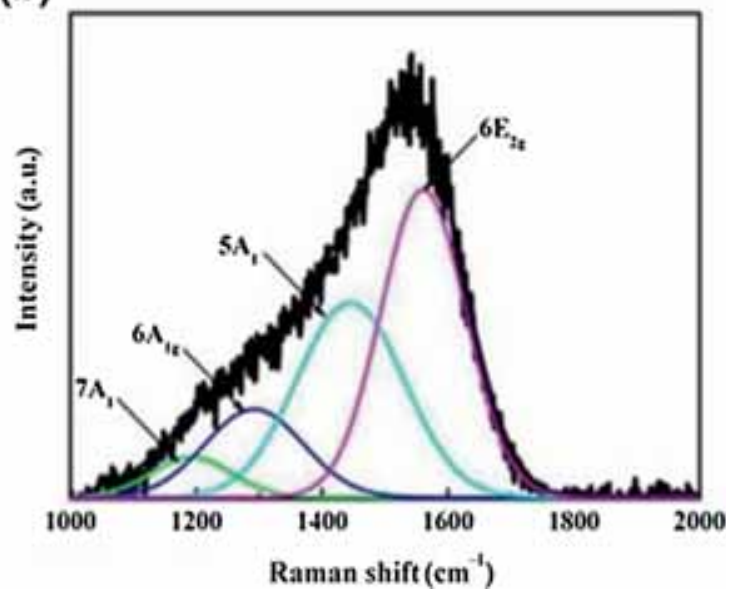

Figure 3. (a) Raman spectra of different samples and (b) the fitted Raman spectra of sample M2.

and chains, while the D peak is attributed to the breathing modes of $s p^{2}$ atoms only in aromatic rings [21]. The Raman spectra of the FL-C:H films can be well fitted by adding two extra peaks at 1260 and $1470 \mathrm{~cm}^{-1}$ which can be attributed to the bent and cross-linked graphitic structures $[9,21,22]$. Therefore, the Raman spectrum of sample M2 (figure 3b) was fitted by four vibrational bands at 1260, 1383, 1470 and $1560 \mathrm{~cm}^{-1}$, corresponding to the $A$-type symmetry of five-, six- and seven-membered rings and $E$-type symmetry of six-membered rings, respectively [22]. Furthermore, the fractional contribution of each vibrational frequency to the Raman spectra is about 0.40 for each of the samples. For pure a-C nanocomposite structures, the high odd ring fraction induces the graphite clusters curvature and interlinking, which are the key to fullerene-like structure formation [23]. The Raman spectra, in accordance with the HRTEM images, indicate the successful synthesis of the FL structure. 


\subsection{Mechanical properties}

Figure 4 displays the compressive stress histogram of the FL$\mathrm{C}: \mathrm{H}$ films. The sample $\mathrm{S} 1$ exhibits lower compressive stress than sample S2. This can be attributed to the fact that during the process of DLC, the films accumulate more heat as the film thickness increases, which leads to higher compressive stress [20]. Besides the film thickness, the compressive stress is mainly determined by the structures of films. When compared with the monolayer films, the multilayer films exhibit lower compressive stress, because the grain growth of one layer is restrained when another layer is deposited onto it, which can lead to stress release during the compressive test [24]. It can also be observed that M2 has the lowest compressive stress among the multilayer samples. That is because multilayer films design, which sets middle transition layers, evidently reduces the compressive stress based on increasing the interface's number. It is beneficial to absorb more energy from the hard layers so as to lower compressive stress in the multilayer films [25].

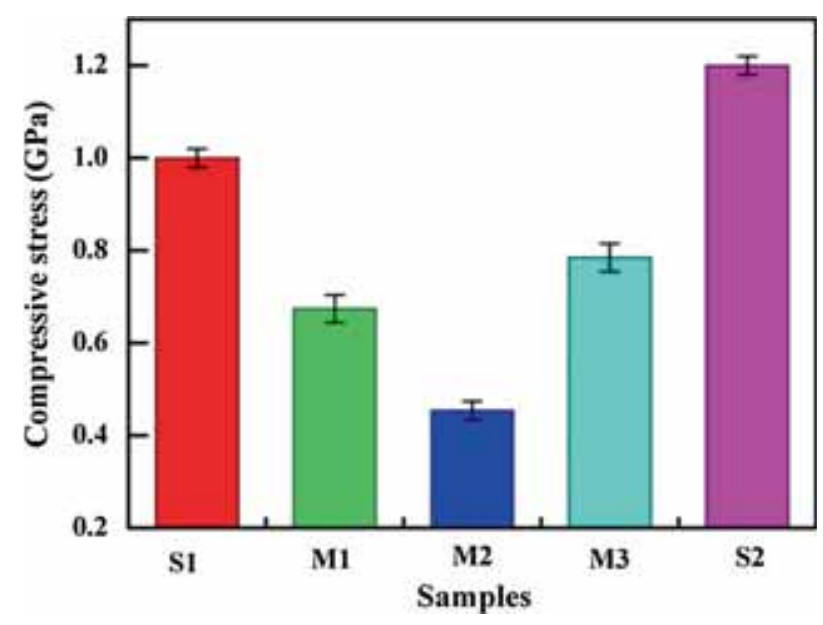

Figure 4. Compressive stress histogram of as-deposited films.

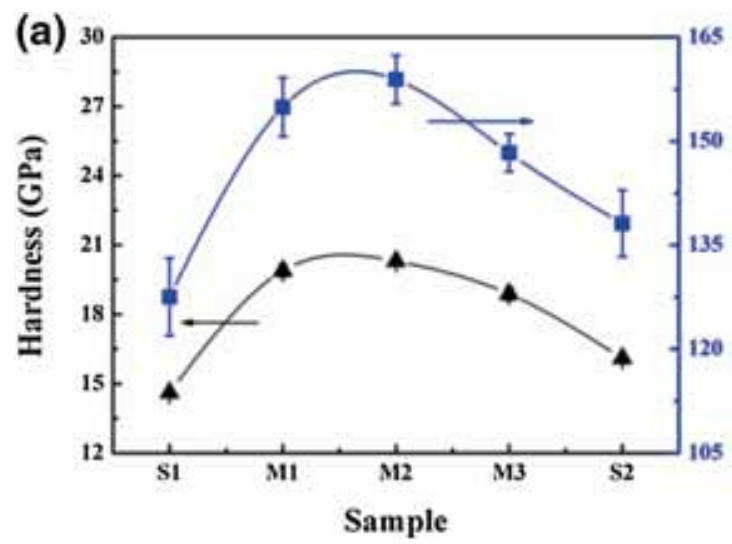

The importance of using multilayer structure films in establishing the excellent mechanical properties are demonstrated in figure 5. The results of mechanical tests are shown in figure 5. Owing to the FL structure, all the FL films show higher hardness and electric modulus than amorphous carbon film and the mechanical properties increase as the content of FL structure increases in the film (figure 5a) [26-28]. The hardness of multilayer films is in the range of 18-21 GPa. The highest hardness (21 GPa) and elastic modulus (160 GPa) are observed for sample M2. Owing to the introduction of a large number of interfaces, which can lead to the hardening effect, the multilayer films all showed higher hardness and elastic modulus than monolayer films [29]. As shown in figure $5 \mathrm{~b}$, the elastic recovery and hardness have same trends and all samples are present in the high elastic recovery value from 84 to $90 \%$. Moreover, the monolayer FL-C:H films have lower elastic recovery than the multilayer FL-C:H films.

\subsection{Tribological performance}

Figure 6 shows the tribological curves and wear rate of the films. Three trials were carried out for each sample. The friction co-efficients and wear rates of the films changed in the same tendency. The lowest friction coefficient $(0.019)$ and wear rate $\left(3.0 \times 10^{-9} \mathrm{~mm}^{3} \mathrm{Nm}^{-1}\right)$ could be obtained for M2 film. The three-dimensional images of the wear tracks and cross section profile of films were shown in figure 7. After 30 min friction test, the width of wear tracks for all samples is about $100 \mu \mathrm{m}$ and the depth of wear tracks for the samples S1, M1, M2, M3 and S2 were about 250, 180, 100, 150 and $200 \mathrm{~nm}$, respectively. As the FL nanostructure can significantly enhance the stability and provide lubrication in humid environment, the multilayer films showed excellent friction properties [4,30,31]. Moreover, multilayer films with more FL structures, which showed excellent hardness and elastic recovery, can significantly improve the stability and deformation recovery of the film, thus resulting in a low wear rate.

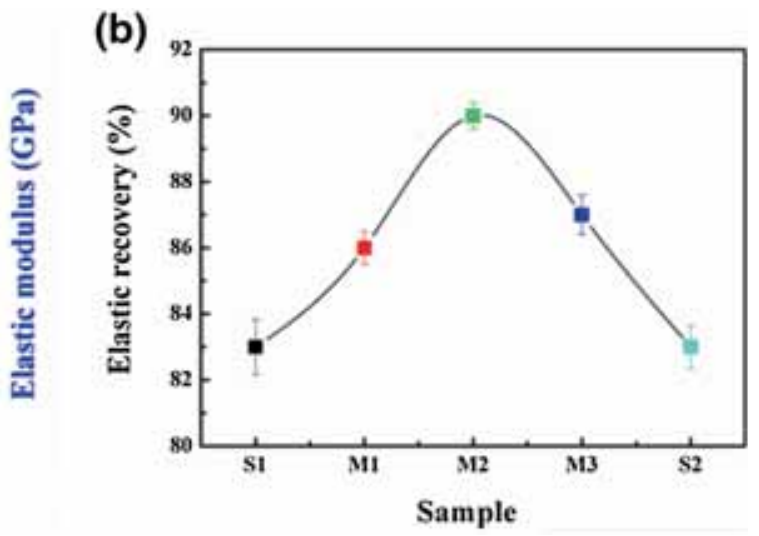

Figure 5. (a) Hardness, elastic modulus and (b) elastic recovery of the samples. 
(a)

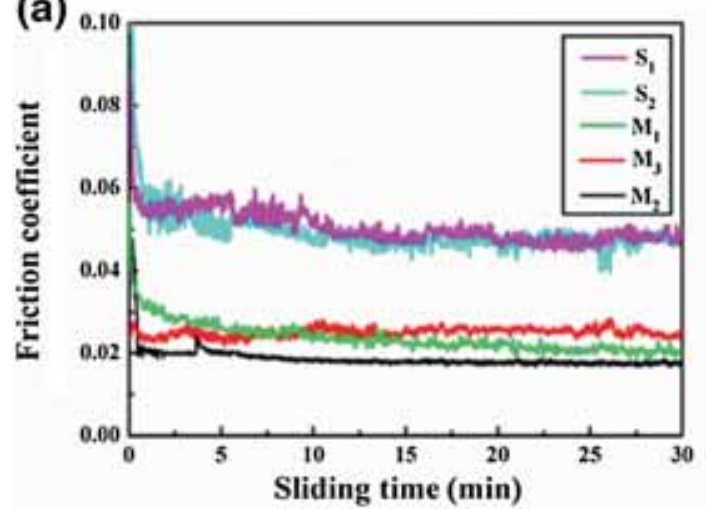

(b)

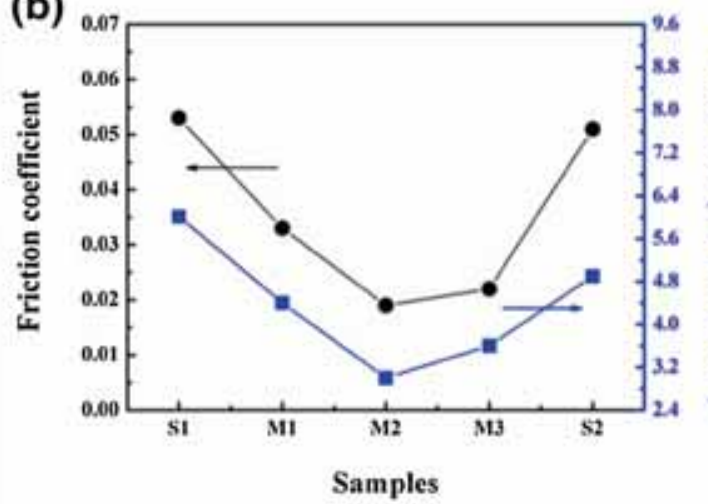

Figure 6. (a) Friction coefficient curves and (b) the average friction coefficient and wear rate of samples.
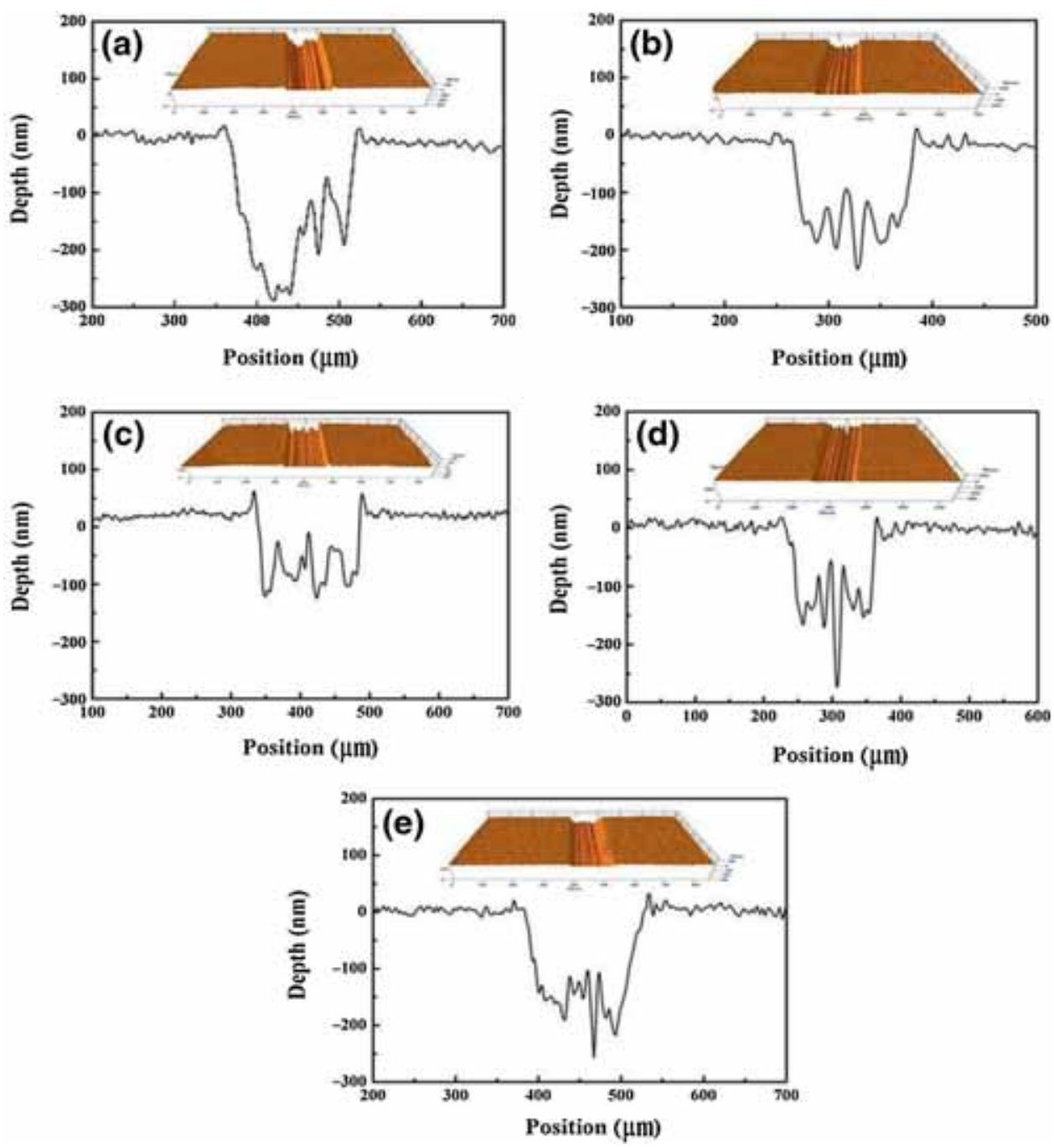

Figure 7. The $3 \mathrm{D}$ images and cross-section profile of the wear tracks of the samples: (a) S1, (b) M1, (c) M2, (d) M3 and (e) S2. 


\section{Conclusions}

The multilayer FL-C:H films with an elastic recovery of $90 \%$ and a friction coefficient of 0.019 were synthesized by using the pulse (pulse-PECVD). Owing to the synergic effect of multilayered and FL structure, the multilayer film exhibited lower compressive stress $(0.45 \mathrm{GPa})$, lower wear rate $\left(3.0 \times 10^{-9} \mathrm{~mm}^{3} \mathrm{Nm}^{-1}\right)$ and higher elastic modulus (160 GPa) when compared to the monolayer films. Owing to the facile preparation, remarkable hardness and ultra-low friction coefficient, the multilayer FL-C:H film has promising applications as super-lubrication material in the industry.

\section{Acknowledgements}

We are grateful to the 973 programme 2013CB632300 and the National Natural Science Foundation of China (Grant no. 51364024, 51275508, 51404124) for their financial support.

\section{References}

[1] Amaratunga G A J, Chhowalla M, Kiely C J, Alexandrou I, Aharonov R and Devenish R M 1996 Nature 383321

[2] Alexandrou I, Kiely C J, Papworth A J and Amaratunga G A J 2004 Carbon $\mathbf{4 2} 1651$

[3] Liu D G, Tu J P, Gu C D, Hong C F, Chen R and Yang W S 2010 Surf. Coat. Tech. 2052474

[4] Gago R, Neidhardt J, Vinnichenko M, Kreissig U, Czigány Z, Kolitsch A et al 2005 Thin Solid Films 48389

[5] Wang Q, Wang C, Wang Z, Zhang J and He D 2007 Appl. Phys. Lett. 91141902

[6] Wang C, Yang S, Wang Q, Wang Z and Zhang J 2008 Nanotechnol. 19225709

[7] Cumings J and Zettl A 2000 Science 289602

[8] Wang P, Wang X, Zhang B and Liu W M 2010 Thin Solid Films 5185938

[9] Wang Q, He D Y, Wang C B, Wang Z and Zhang J Y 2008 J. Appl. Phys. 104043511
[10] Buijnsters J G, Camero M, Vazquez L, Agullo-Rueda F, Gago R, Jimenez I et al 2010 Diam. Relat. Mater. 191093

[11] Wang P, Wang X, Liu W and Zhang J 2008 J. Phys. D Appl. Phys. 4168

[12] Wang X, Wang P, Yang S and Zhang J 2008 Wear 265 1708

[13] Dwivedi N, Kumar S, Ishpal, Dayal S, Govind, Rauthan C M S et al 2011 J. Alloys Compd. 5091285

[14] Wu Y, Li H, Ji L, Ye Y, Chen J and Zhou H 2013 Surf. Coat. Tech. 236438

[15] Shi J, Cao Z H, Wei M Z, Pan G, Xu L J and Meng X K 2014 Mat. Sci. Eng. A-Struct. 618385

[16] Mori T, Noborisaka M, Watanabe T and Suzuki T 2012 Surf. Coat. Tech. 213216

[17] Guo Y B, Wang D G and Zhang S W 2011 Tribol. Int. 44 906

[18] Gao X M, Hu M, Sun J Y, Fu Y L, Yang J, Liu W M et al 2015 Appl. Surf. Sci. $\mathbf{3 3 0} 30$

[19] Donnet C and Erdemir A 2008 Tribology of diamond-like carbon films (New York, US: Springer)

[20] Neidhardt J, Hultman L and Czigány Z 2004 Carbon 422729

[21] Wang Q, Wang C B, Wang Z, Zhang J Y and He D Y 2008 Appl. Phys. Lett. 93131920

[22] Siegal M P, Tallant D R, Martinez-miranda L J, Barbour J C, Simpson R L and Overmyer D L 2000 Phys. Rev. B 61 10451

[23] Martınez-Miranda L J, Siegal M P and Provencio P P 2001 Appl. Phys. Lett. 79542

[24] Ban M, Hasegawa T, Fujii S and Fujioka J 2003 Diam. Relat. Mater. 1247

[25] Pujada B R and Janssen G C A M 2006 Surf. Coat. Tech. 201 4284

[26] Flores-Ruiz F J, Enriquez-Flores C I, Chinas-Castillo F and Espinoza-Beltran F J 2014 Appl. Surf. Sci. 314193

[27] Hellgren N, Johansson M P, Broitman E, Hultman L and Sundgren J E 1999 Phys. Rev. B 595162

[28] Neidhardt J and Hultman L 2007 J. Vac. Sci. Technol. A 25633

[29] Cruz R, Rao J, Rose T, Lawson K and Nicholls J R 2006 Diam. Relat. Mater. 152055

[30] Ji L, Li H, Zhao F, Quan W, Chen J and Zhou H 2009 J. Appl. Phys. 105106113

[31] Liu X Q, Yang J, Hao J Y, Zheng J Y, Gong Q Y and Liu W M 2012 Adv. Mater. 244614 